\title{
Position Paper
}

\section{Decreased clinical response to therapy in pediatric patients with cerebral palsy: Current trends and challenges}

\author{
Matthew McLaughlin ${ }^{\mathrm{a}, *}$ and Didem Inanoglu ${ }^{\mathrm{b}}$ \\ ${ }^{a}$ University of Missouri-Kansas City School of Medicine, Children's Mercy - Kansas City, Kansas City, MO, USA \\ ${ }^{\mathrm{b}}$ University of Texas Southwestern Medical Center, Dallas, TX, USA
}

Keywords: Clinical response, pediatric patients, cerebral palsy

\section{Scenario}

The patient is a 7-year-old male with diplegic cerebral palsy (CP) Gross Motor Functioning Classification System (GMFCS) III. He ambulates with bilateral solid ankle foot orthoses (AFOs). For longer distances he uses a reverse walker, and for shorter distances he can walk independently but has difficulty with unlevel surfaces and stairs. He is previously known to have a jump gait pattern with increased tone in bilateral hip adductors, knee flexors, and ankle plantar flexors. Lately he has started to crouch more, have more mid-foot collapse and toe outwards.

He has been in your care since his diagnosis of cerebral palsy at 2 years old. He has been wearing braces since that time. He receives outpatient physical therapy weekly, and he does his home exercise program a few times a week. Cognitively he is age appropriate, and his speech is typical. He is continent of bowel and bladder, and his constipation is managed with daily polyethylene glycol. He does not have any complaints of pain, but his parents are worried about his legs.

${ }^{*}$ Corresponding author: Matthew McLaughlin, University of Missouri Kansas City, Kansas City, MO, USA. E-mail: mjmclaughlin@ cmh.edu.
Orthopedically, he does not have significant scoliosis and his hips are showing mild hip dysplasia with a lateral migration of $10 \%$ bilaterally.

$\mathrm{He}$ has received botulinum toxin injections by the same provider since age 2 . At first the injections were every 6 months and recently have been every 3 months. His family is reporting that the injections are still helpful, but they don't last as long as they use to last.

\section{Dr. McLaughlin}

Within the heterogeneous group that is pediatric patients with $\mathrm{CP}$, those that fall into the GMFCS III category present the most challenges and opportunities from a treatment perspective. Depending on the individual child and their age, maximizing oral medications, chemodenervation injections, selective dorsal rhizotomies, orthopedic surgery, and intrathecal baclofen pumps are all potential treatment options. Administration of botulinum toxin (BoNT) type A in children with $\mathrm{CP}$ results in statistically significant spasticity reduction at 2 weeks, 4 weeks, and more than 3 months after treatment [1-3]. Although this case stresses the need for continued follow-up after BoNT injections to observe independent measures of clinical responses, it appears 
this patient has decreased clinical responses to repetitive injections. Decreasing clinical responses can be related to several different factors.

As this patient has grown, it may be that the dosage of BoNT has not changed, and realistically he may have received a relatively lower unit of BoNT per kilogram injected. The manufacturer of onabotulinum toxin A has a dosing limit of 340 units with a maximum of 10 units/kg [4]. For a spastic diplegic patient at the age of 7 whose weight is at the $50^{\text {th }}$ percentile $(26 \mathrm{~kg})$, maximum dosing would be roughly 260 units. Based on a survey of pediatric physiatrists in the United States, most prefer to limit the total body dose to 400 units $(39.7 \%)$, while the second and third most common dose limits were $500(21.2 \%)$ and $600(20.2 \%)$ units [5]. The dosage on the drug package insert can vary significantly from what is commonly used in clinical practice. Reviewing the amount of injected units may provide a little more detail about why the family is noting diminishing responses with subsequent injections. In addition to the total body dose used, attention should be given to the amount injected into each muscle group. Appropriate ranges for BoNT per muscle group are provided as guidelines by manufacturers. The reason for reduced clinical response may be the distribution of the total amount into the individually and correctly selected muscle groups versus the total body dose injected.

Another potential reason for the decreased clinical response is the frequency of injections. BoNT is most effective in muscle that is non-fibrotic and has receptor sites that are available for the toxin to block [6]. Rats who received high-dose BoNT injections have exhibited increased muscle atrophy and decreased muscle volume [6]. As muscle volume and architecture is highly correlated with muscle strength in children with $\mathrm{CP}$, [7] loss of muscle volume and strength could result in diminishing positive responses from the family. Recently, more studies have demonstrated diminishing benefits with subsequent injections at short intervals [8]. Furthermore, equivalent clinical responses for patients who receive injections at intervals of either every 4 months or yearly were the same in children that were using abobotulinum toxin A [9]. Of note, this study was not in an American cohort of children and the authors used a more distal BoNT site, possibly changing the effectiveness of the medical intervention. Therefore, extrapolation of those results needs to be evaluated in the larger context of the additional care those patients received. Also, it is crucial to differentiate a fixed contracture from increased muscle tone. One way of doing this is an exam under general anesthesia at the time of injec- tion. A technique utilized in adult patients who had a cerebrovascular accident is to perform a nerve block in order to confirm a muscle tone issue; however, this technique may not translate to pediatric patients with CP [10].

While the family is reporting decreased clinical response with onabotulinum toxin A, it does not appear this patient has a history of being prescribed oral medications to help with spasticity. Children with $\mathrm{CP}$ have shown reduction in tone after being prescribed oral baclofen [11] or tizanidine [12]. Furthermore, in pediatric patients with $\mathrm{CP}$ who received onabotulinum toxin A injections with concomitant oral tizanidine therapy, there was a significant improvement over the group receiving onabotulinum toxin A injections and oral baclofen [12]. While this could represent a theoretical synergistic action between the two medications, it likely represents the benefit of targeting two different mechanisms of action to decrease tone, with tizanidine acting centrally and onabotulinum toxin A acting peripherally. In certain patients, optimal treatment of spasticity or a movement disorder may be optimized with a combination of BoNT with a concomitantly administered oral medication, such as baclofen, tizanidine, diazepam, or dantrolene [13]. Each individual patient may respond differently to each oral medication as their tone may be impacted differently based on inhibition of a certain neurotransmitter.

A significant challenge in treating pediatric patients with $\mathrm{CP}$ is ensuring we are optimizing the right dose of the right medication at the right time for the right patient. These concepts are referred to frequently when implementing precision medicine principles for treating patients. The increasing shift to precision medicine is embodied by proactive medication and dosage selection using evidence-based predictions for drug response or adverse events [14]. Genetic variation represents one source of inter-individual variability, and thus pharmacogenomic approaches are expected to make substantial contributions to medicine and hold the promise to transform the development, labeling, and use of therapeutic agents $[15,16]$. In pediatric patients with $\mathrm{CP}$, a small pharmacogenetic study reported differences in response to oral spasticity treatments based on the genes of each individual patient [11]. While aspects of incorporation of precision medicine have been more routinely described in oral medication such as baclofen which require genes to encode transporters for absorption, distribution, metabolism, and elimination of this drug, [17] injectable toxins bypass many of these processes as the medication is delivered locally into the muscle tissue. 
A potential example of incorporating aspects of precision medicine in a patient with $\mathrm{CP}$ would be to evaluate the antibody production to potentially determine whether to inject with a certain BoNT. The presence of antibodies appears to be increased in patients who receive higher injected doses and who have received injections for a longer duration [18]. Presumably, this patient has been injected with onabotulinum toxin A. Switching to rimabotulinum toxin B may provide some benefit in patients who were non-responsive to prior injections; however, more than $25 \%$ of patients remained unresponsive to rimabotulinum toxin B injections [19].

\section{Dr. Inanoglu}

In children with $\mathrm{CP}$, gait abnormalities may be complex and very difficult to characterize clinically. In the literature there are different gait classification systems developed to analyze gait deviations. Current consensus evidence describes six multiple joint patterns in attempts to identify underlying pathophysiology and inform decision making for treatment [20].

In this patient's case an instrumental gait analysis (IGA) may be the next best step to demonstrate the underlying pathological processes, distinguish between different levels of impairment, and develop a treatment plan as well as monitor progress. IGA can enhance the likelihood of positive outcomes and reduce the number of negative outcomes [21]. In evaluation of this child with ambulatory $\mathrm{CP}$, the first step is to confirm which of the six joint patterns are present and to what extent: genu recurvatum, drop foot, true equinus, jump gait, apparent equinus or crouch gait. This in return will help to determine the cause of each deviation, e.g., out toeing could be from multiple causes like pes plano valgus and external hip rotation [22]. These findings should be carefully considered prior to non-surgical or surgical treatment to allow all sites of pathology to be addressed and to optimize outcomes.

In the development of the next steps of care for this patient, it is important to set short-term and long-term treatment goals that are specific, measurable, achievable, realistic and time based. The family-centered care model emphasizes the role of parents in the goal setting. The key elements of family-centered practice include an emphasis on child and family strengths rather than deficits, facilitating family choice and control, and creating a therapeutic environment that optimizes the development of a collaborative relationship. To engage families and support their involvement in rehabilita- tion services, it is important for providers to serve as collaborators, consultants, facilitators, educators, and coaches [23].

The other critical aspect of this ambulatory patient's care is gait rehabilitation. Physical therapists (PTs) are an important part of the multidisciplinary care team. Current literature shows that trunk control, not the traditionally claimed spasticity, may be the strongest predictor for gait capacity in children with CP (GMFCS I-III) and that leg muscle strength and selective voluntary control are strongly related to trunk control in this cohort [24]. The child's physical therapy plan should be revisited and should include functional electrical stimulation (FES) [25], robotic training, and aquatics (if available).

At International Classification of Function (ICF) by the World Health Organization body structure and function level, there is evidence that FES may increase (active) ankle dorsiflexion angle and strength and improve selective motor control, balance, and gait kinematics [26]. Robotic training also provides benefits for patients with $\mathrm{CP}$, specifically by increasing walking speed and endurance and improving gross motor function [27]. Another helpful therapy approach is aquatics. In 2015, Lai et al. [28] showed that a pediatric aquatic therapy program improved gross motor function measured by the 66-item Gross Motor Function Measure (GMFM-66) and generated greater enjoyment than conventional therapy. Aquatic therapy exerts both thermal and mechanical effects that enhance motor function. The thermal effects are beneficial for pain and spasticity reduction. The mechanical effects provide benefits by decreasing the influence of gravity and joint loading and facilitating postural support and muscular strength.

In this patient with $\mathrm{CP}$, hippotherapy may also be considered for the specific goal of improved trunk posture control [29]. Current evidence shows hippotherapy may be useful to maximize the functional performance of children with $\mathrm{CP}$ when outcome measures such as GMFM-66 and Pediatric Evaluation of Disability Inventory-Functional Skills Scale (PEDI-FSS) are used [30]. GMFM-66 measures the child's capacity for gross motor function in a standardized environment, while the PEDI-FSS measures performance in functional activities of daily life. When used together, the GMFM and PEDI-FSS may provide a comprehensive picture of a child's functional abilities at the ICF activities level [31]. With the use of hippotherapy, significant gains have been observed in walking, running and jumping. The GMFM-66 represents the unidimensional construct of gross motor ability according to task diffi- 
culty and thus is recommended for research purposes when comparing changes in gross motor function over time in children with CP [32].

The best model of care for spasticity and abnormal gait is provided by a multidisciplinary team using a multimodal approach. In this child's care, consulting with the team that ideally includes a pediatric orthopedic surgeon and a PT would help outline the underlying complex deformities and abnormal movement patterns. Even if there is no opportunity for an IGA, an observational gait analysis through a video recording by the multidisciplinary spasticity team would provide better insight into the pathophysiology [33]. Other team members may include a pediatric neurologist who is specialized in movement disorders and a neurosurgeon.

Finally, in the multidisciplinary consultation rounds, different treatment options would be discussed based on the goals determined and the data obtained. These potential care options include oral anti-spasticity medications, chemodenervation with serial casting, phenol/alcohol motor point or nerve blocks, orthopedic surgery, intrathecal baclofen therapy and selective dorsal rhizotomy.

\section{Updated case}

Since you last saw your patient, he is now an 8 year old. He did a gait study at your local gait lab, and this evaluation was interpreted by an orthopedic surgeon, a fellow pediatric rehabilitation medicine physician and a pediatric PT. They recommended an intensive outpatient physical therapy program. He went through this program and, at first, he was walking longer distances with greater efficiency of movement. He reported less fatigue and an overall sense of feeling stronger. Eight months later he reported that his spasticity had worsened and he was not walking as long or as far. He did another intensive outpatient therapy program which did not improve his walking speed or efficiency of movement as much as his first round of intensive therapies. The family tries to do a robust home-exercise program but is limited by family constraints. He presents to your clinic with his family, who asks, "What else can we do to get his walking better?", "Is there something more besides surgery that could improve his walking?", "BoNT injections in the past were helpful; could we try that again?", and "Is there something wrong with his muscles because it keeps getting harder to walk over time?".

\section{Dr. Inanoglu}

This would be a good opportunity to revisit the natural course of $\mathrm{CP}$ with this patient and family. Children with $\mathrm{CP}$ reach a plateau in gross motor skills by 6-7 years of age [34] and there is a measurable deterioration in their gait when they reach their teenage years if there is no intervention [35]. Gait is most inefficient at age 12 in ambulatory children with $\mathrm{CP}$ and gross motor function skills deteriorate shortly afterwards [36]. The walking velocity decreases, they spend more time in double support, their stiffness increases in hips and knees, and a gradual shift is seen from equinus through neutral to calcaneus at the ankles [37]. Furthermore, $10-20 \%$ become non-ambulatory by age 40 [35]. With this slow downward trajectory, maintenance of the gait of this child at the current level is still an improvement over natural history. Children with GMFCS III and IV are at the greatest risk for this decline in function [35]. Current knowledge indicates decreased physical activity, decreased participation in physical therapy and fitness programs, loss of strength, contractures and pain are the most common factors for functional decline in individuals with CP [35]. Over 30 years of clinical research have demonstrated that leisure-time physical activity participation is critical for enhancing health, function, and well-being in children and adults with CP [38-40]. Thus maintaining a fitness and exercise program is crucial for this patient in this case.

In addition, even though the underlying injury is static in CP, the manifestations may evolve over time. Children may go through different phases with their tone issues before they end up with abnormal motor control and movement patterns as late as the second decade of life [35]. Injury to specific brain centers generates specific types of functional loss; however, in the end this cohort of children has three primary abnormalities of gait: loss of selective motor control, impaired balance, and abnormal tone. Furthermore, secondary musculoskeletal abnormalities emerge slowly over time in direct proportion to the rate of skeletal growth [35]. A spastic muscle will eventually develop true contracture as muscle growth fails to keep pace with bone growth. The distal biarticular muscles are usually the most severely involved and most prone to contractures. Bones also fail to mold or remodel normally as they grow, leading to rotational deformities. As is the case with this patient, pathological gait is complex and can result from multiple etiologies. For example, crouch gait can be secondary to poor balance, loss of selective motor control, abnormal tone, weakness, and lever-arm 
dysfunction. At the same time there may be muscle fiber atrophy and fiber loss resulting in shortening of muscles and reduced muscle volumes [41].

The history of functional decline for this 8-year-old patient also necessitates a comprehensive neurological exam to assess for any potential new focal deficits and to identify the existing component of dystonia, with cocontraction of muscles across joints contributing to positional abnormalities. A detailed musculoskeletal exam can reveal pelvic tilt and femoral or tibial torsion that can be addressed in physical therapy with de-rotational straps. If there has been any metabolic or energy assessment during IGA showing increased $\mathrm{O} 2$ consumption, OT referral to address energy conservation and instrumental ADL training would be helpful. Optimizing use of assistive and adoptive devices and orthotics can make a difference. A custom hinged AFO may be considered in this case to provide support in mid-stance and energy return in push-off augmenting power generation at the ankle [42].

With this patient who reports decreasing activity levels and endurance, it is also important to consider other comorbidities such as seizures, mood disorders, insomnia, anemia, obesity, failure to thrive and pain as potential contributors to functional decline. Addressing these medical conditions with appropriate evaluations may be needed. Repeating brain MRI for worsening seizures, consulting neuropsychology for mood and cognitive evaluation, referring to a sleep team or sleep study for possible sleep disordered breathing, ordering blood work for anemia and poor nutrition or consulting with a nutritionist for increased weight gain may all potentially contribute to improved function. Pain is a common risk factor in growing children with CP [43]. Imaging studies may help localize the source of pain if joint or bone abnormalities, such as hip dislocation, are suspected. Consulting with a multidisciplinary pain team may be beneficial for diagnosis of the etiology of pain as well as providing management.

As mentioned earlier, the best approach to a complex gait problem is to break it down into individual components that can be studied by focused physical examination, imaging, and gait analysis. Once a specific and accurate problem list is developed, addressing each condition individually is the next step in management. In this patient's case, if there is worsening spasticity or dystonia, resuming BoNT may still be a reasonable approach. The type or the brand of BoNT used in the past may be changed for the next injection cycle since the diffusion to adjacent and remote muscles of different toxins may be different due to their protein com- position [44]. Muscle selection should reflect current specific problems and target realistic goals agreed on by the family and patient. Also prioritizing the antagonistic muscles that show exacerbated co-contraction may be a better approach in muscle selection [45]. For each muscle selected, the total body units may be increased based on the current weight of the patient and the size of the muscle. The possibility of resistance and using precision medicine principles are all important parts of the discussion at this time and should be reviewed with the family. Performing the injection under general anesthesia or doing selective nerve blocks should be considered to differentiate fixed contractures from muscle hypertonia. Phenol or alcohol blocks can be combined with BoNT to spare the neurotoxin for widespread use and provide longer lasting relief in selected areas. Follow up care after BoNT with serial casting may help with equinovarus deformities. As discussed previously, systemic medications such as baclofen, tizanidine, etc., can be used in conjunction with injectables with close monitoring for side effects such as cognition, vision, bladder and bowel function. Medical use of cannabis or cannabidiol oil through compassionate cultivation may also be considered. Even though the evidence is controversial for the use in spasticity $[46,47]$, Compassionate use means expanded access to investigational drugs for treatment of serious or life-threatening illness outside of clinical trials when no comparable or satisfactory option is available. State Medical Board Registries may guide physicians in the appropriate use and regulation of dosage.

Finally, if there is no progress seen with any of these interventions, repeating the IGA to develop an accurate and precise problem list to inform surgical decision making is a good approach. Potential surgical options include single event multilevel surgery, selective dorsal rhizotomy or intrathecal baclofen and can be reviewed by the multidisciplinary spasticity team.

\section{Dr. McLaughlin}

As Dr. Inanoglu referenced, although CP is a static central injury, it has many peripheral manifestations. As it has been a longer time since his last BoNT injections, resuming this intervention may provide a more optimal clinical outcome than prior injections. This 'drug holiday' has been shown to provide beneficial outcomes after a long duration of intrathecal baclofen treatment, for example [10]. After drug holidays, studies have shown decreased adverse effects and improved or sim- 
ilar levels of drug response at lower doses with medications commonly used by or familiar to physiatrists such as intrathecal baclofen [48], levodopa [49], and methylphenidate [50]. Drug holidays allow for evaluation of efficacy and tolerability. Temporary cessation of the medication may provide information about whether the medication was actually useful or not [51]. In the case of this specific patient, it may have allowed for improvement in the muscle fibers that were present. Because BoNT irreversibly binds at the pre-synaptic terminal, a drug holiday may theoretically allow for a healthier nerve terminal. Unfortunately, drug holidays are not without risk. In the case of a medication like intrathecal baclofen, rapid downward titration for a drug holiday may result in withdrawal symptoms which can be life-threatening. Fortunately for this patient, there are no withdrawal symptoms for cessation of BoNT. However, there is a risk for more spastic muscles, alterations in gait, or potential contracture formations if BoNT was beneficial. Weighing the risk and benefits of and optimally timing a drug holiday, may be advantageous to both providers and patients by allowing evaluations to occur without specific interventions.

Directly addressing the question of "Is there something wrong with his muscles because it keeps getting harder to walk over time?" involves explaining that muscle architecture is directly related to muscle function in pediatric patients with CP [52]. Several factors could be contributing to a potential change in this patient's muscle architecture that have changed the apparent gait pattern. Muscle architecture may have changed with repetitive BoNT injections, as this has been shown to cause fibrillar and non-fibrillar changes in non-human studies [6]. Characterizing these changes specific to pediatric patients with $\mathrm{CP}$ becomes a little more challenging. Additionally, the prolonged time since this patient's last treatment or the diminishing clinical response observed over time may have caused a change within this patient's muscle development [9].

To help address this patient's other questions, it certainly seems reasonable that the resumption of BoNT injections would be a potential treatment option. The family seems hesitant to try surgery, but there is also the option of oral medications. Tizanidine and baclofen have been used as adjunctive treatments to BoNT in the past with relatively small numbers of adverse effects [53]. Additionally, if the IGA demonstrates primarily spasticity in the absence of dystonia and good motor control, a selective dorsal rhizotomy may be a reasonable suggestion.

Overall, this case is a common problem encountered by physicians treating $\mathrm{CP}$, which highlights the need for continued research into spasticity treatment and management. Although important to note the challenges of this case, this patient is only 8 years old and will likely need many different treatment options during the course of his lifetime. Regardless, the family will look toward their medical team to provide the best option both at the time of the clinical visit and for years to come.

\section{Dr. Inanoglu}

In closing, $\mathrm{CP}$ is a multisystem disorder that results in complex movement abnormalities. Ambulatory children with CP commonly manifest with a multi-joint gait disorder that requires a thorough and multidisciplinary evaluation. In planning the family-centered treatment care, it is important to develop a holistic approach and address different planes of motion at multiple segments of the lower extremities. With the limitations of the current quality evidence, future research should focus on better longer term strategies to successfully prevent worsening of gait and function in this cohort of children.

\section{Conflict of interest}

The authors have no conflicts of interest to report.

\section{References}

[1] Corry IS, Cosgrove AP, Duffy CM, McNeill S, Taylor TC, Graham HK. Botulinum toxin A compared with stretching casts in the treatment of spastic equinus: A randomised prospective trial. J Pediatr Orthop 1998; 18: 304-311.

[2] Mall V, Heinen F, Siebel A, Bertram C, Hafkemeyer U, Wissel J, et al. Treatment of adductor spasticity with BTX-A in children with $\mathrm{CP}$ : A randomized, double-blind, placebocontrolled study. Dev Med Child Neurol. 2006; 48(1): 10-13. doi: 10.1017/S0012162206000041.

[3] Corry IS, Cosgrove AP, Walsh EG, McClean D, Graham HK. Botulinum toxin A in the hemiplegic upper limb: A doubleblind trial. Dev Med Child Neurol. 1997; 39(3): 185-193. doi: 10.1111/j.1469-8749.1997.tb07408.x.

[4] BOTOX (onabotulinumtoxinA) Label - FDA. Available from: https://www.accessdata.fda.gov/drugsatfda_docs/label/2011/ 103000s5236lbl.pdf.

[5] Paulson A, Zigler CK, Houtrow A, Pruitt D. Botulinum toxin: Techniques within pediatric physiatry. PM R. 2019; 11(1): 38-44. doi: 10.1016/j.pmrj.2018.06.004.

[6] Pingel J, Nielsen MS, Lauridsen T, Rix K, Bech M, Alkjaer $\mathrm{T}$, et al. Injection of high dose botulinum-toxin A leads to impaired skeletal muscle function and damage of the fibrilar and non-fibrilar structures. Sci Rep. 2017; 7(1): 14746. doi: 10.1038/s41598-017-14997-3. 
[7] Moreau NG, Simpson KN, Teefey SA, Damiano DL. Muscle architecture predicts maximum strength and is related to activity levels in cerebral palsy. Phys Ther. 2010; 90(11): 1619 1630. doi: 10.2522/ptj.20090377.

[8] Kahraman A, Seyhan K, Deger U, Kutlutürk S, Mutlu A. Should botulinum toxin A injections be repeated in children with cerebral palsy? A systematic review. Dev Med Child Neurol. 2016; 58(9): 910-917. doi: 10.1111/dmcn.13135.

[9] Kanovsky P, Bares M, Severa S, Richardson A. Paediatric Limb Spasticity Study G. Long-term efficacy and tolerability of 4-monthly versus yearly botulinum toxin type A treatment for lower-limb spasticity in children with cerebral palsy. Dev Med Child Neurol. 2009; 51(6): 436-445. doi: 10.1111/j.14698749.2008.03264.x.

[10] Deltombe T, De Wispelaere JF, Gustin T, Jamart J, Hanson P. Selective blocks of the motor nerve branches to the soleus and tibialis posterior muscles in the management of the spastic equinovarus foot. Arch Phys Med Rehabil. 2004; 85(1): 54-58. doi: 10.1016/s0003-9993(03)00405-2.

[11] McLaughlin MJ, He Y, Brunstrom-Hernandez J, Thio LL, Carleton BC, Ross CJD, et al. Pharmacogenomic variability of oral baclofen clearance and clinical response in children with cerebral palsy. PM R. 2018; 10(3): 235-243. doi: 10.1016/j. pmrj.2017.08.441.

[12] Dai AI, Askoy SN, Demirüyek AT. Comparison and side effects of oral baclofen versus tizanidine therapy with adjuvant botulinum toxin type $\mathrm{A}$ in children with cerebral palsy and spastic equinus foot deformity. J Child Neurol. 2016; 31(2): 184-189. doi: 10.1177/0883073815587030.

[13] Delgado MR, Hirtz D, Aisen M, Ashwal S, Fehlings DL McLaughlin J, et al. Practice parameter: Pharmacologic treatment of spasticity in children and adolescents with cerebral palsy (an evidence-based review): Report of the Quality Standards Subcommittee of the American Academy of Neurology and the Practice Committee of the Child Neurology Society. Neurology. 2010; 74(4): 336-343. doi: 10.1212/WNL.0b013e $3181 \mathrm{cbcd} 2$.

[14] Abrahams E, Ginsburg GS, Silver M. The Personalized Medicine Coalition: Goals and strategies. Am J Pharmacogenomics. 2005; 5(6): 345-355. doi: 10.2165/00129785-20050 5060-00002.

[15] Lindpaintner K. Pharmacogenetics and the future of medical practice. Br J Clin Pharmacol. 2002; 54(2): 221-230. doi: 10. 1046/j.1365-2125.2002.01630.x.

[16] Smart A, Martin P. The promise of pharmacogenetics: Assessing the prospects for disease and patient stratification. Stud Hist Philos Biol Biomed Sci. 2006; 37(3): 583-601. doi: 10.1016/j. shpsc.2006.06.002.

[17] McLaughlin MJ, Abdel-Rahman S, Leeder JS. Examining the role of precision medicine with oral baclofen in pediatric patients with cerebral palsy. Curr Phys Med Rehabil Rep. 2019; 7(1): 40-45.

[18] Albrecht P, Jansen A, Lee JI, Moll M, Ringelstein M, Rosenthal D, et al. High prevalence of neutralizing antibodies after long-term botulinum neurotoxin therapy. Neurology. 2019; 92(1): e48-e54. doi: 10.1212/WNL.0000000000006688.

[19] Walter U, Mühlenhoff C, Benecke R, Dressler D, Mix E, Alt J, et al. Frequency and risk factors of antibody-induced secondary failure of botulinum neurotoxin therapy. Neurology. 2020; 94(20): e2109-e2120. doi: 10.1212/WNL.0000000000009444.

[20] Papageorgiou E, Nieuwenhuys A, Vandekerckhove I, Van Campenhout A, Ortibus E, Desloovere K. Systematic review on gait classifications in children with cerebral palsy: An up- date. Gait Posture. 2019; 69: 209-223. doi: 10.1016/j.gaitpost. 2019.01.038.

[21] Dugan EL, Shilt JS. The role of motion analysis in surgica planning for gait abnormalities in cerebral palsy. Phys Med Rehabil Clin N Am. 2020; 31(1): 107-115. doi: 10.1016/j.pmr. 2019.09.009

[22] Cao LA, Rethlefsen SA, Wren TAL, Kay RM. Causes of outtoeing gait in children with cerebral palsy. Gait Posture. 2020; 76: 141-145. doi: 10.1016/j.gaitpost.2019.12.002.

[23] King G, Chiarello L. Family-centered care for children with cerebral palsy: Conceptual and practical considerations to advance care and practice. J Child Neurol. 2014; 29(8): 10461054. doi: 10.1177/0883073814533009.

[24] Balzer J, Marsico P, Mitteregger E, van der Linden ML, Mercer TH, van Hedel HJA. Influence of trunk control and lower extremity impairments on gait capacity in children with cerebral palsy. Disabil Rehabil. 2018; 40(26): 3164-3170. doi: 10 1080/09638288.2017.1380719.

[25] Karabay I, Dogan A, Dogan Arslan M, Dost G, Ozgirgin N Effects of functional electrical stimulation on trunk control in children with diplegic cerebral palsy. Disabil Rehabil. 2012; 34(11): 965-970. doi: 10.3109/09638288.2011.628741.

[26] Moll I, Vles JSH, Soudant DLHM, Witlox AMA, Staal HM, Speth LAWM, et al. Functional electrical stimulation of the ankle dorsiflexors during walking in spastic cerebral palsy: A systematic review. Dev Med Child Neurol. 2017; 59(12): 1230-1236. doi: 10.1111/dmcn. 13501.

[27] Carvalho I, Medeiros Pinto S, das Virgens Chagas D, Praxedes Dos Santos JL, de Sousa Oliveira T, Alberto Batista L. Robotic gait training for individuals with cerebral palsy: A systematic review and meta-analysis. Arch Phys Med Rehabil. 2017; 98(11): 2332-2344. doi: 10.1016/j.apmr.2017.06.018.

[28] Lai CJ, Liu WY, Yang TF, Chen CL, Wu CY, Chan RC. Pediatric aquatic therapy on motor function and enjoyment in children diagnosed with cerebral palsy of various motor severities. J Child Neurol. 2015; 30(2): 200-208. doi: 10.1177/ 0883073814535491.

[29] Martín-Valero R, Vega-Ballón J, Perez-Cabezas V. Benefits of hippotherapy in children with cerebral palsy: A narrative review. Eur J Paediatr Neurol. 2018; 22(6): 1150-1160. doi: 10.1016/j.ejpn.2018.07.002.

[30] Park ES, Rha DW, Shin JS, Kim S, Jung S. Effects of hippotherapy on gross motor function and functional performance of children with cerebral palsy. Yonsei Med J. 2014; 55(6): 1736-1742. doi: 10.3349/ymj.2014.55.6.1736.

[31] Ketelaar M, Vermeer A, Helders PJ. Functional motor abilities of children with cerebral palsy: A systematic literature review of assessment measures. Clin Rehabil. 1998; 12(5): 369-380. doi: 10.1191/026921598673571117.

[32] Russel DJ, Avery LM, Rosenbaum PL, Raina PS, Walter SD, Palisano RJ. Improved scaling of the gross motor function measure for children with cerebral palsy: Evidence of reliability and validity. Phys Ther. 2000; 80(9): 873-885.

[33] Rathinam C, Bateman A, Peirson J, Skinner J. Observational gait assessment tools in paediatrics-a systematic review. Gait Posture. 2014; 40(2): 279-285. doi: 10.1016/j.gaitpost. 2014.04.187.

[34] Rosenbaum PL, Walter SD, Hanna SE, Palisano RJ, Russell DJ, Raina $\mathrm{P}$, et al. Prognosis for gross motor function in cerebral palsy: Creation of motor development curves. JAMA. 2002; 288(11): 1357-1363. doi: 10.1001/jama.288.11.1357.

[35] Gage JR, Schwartz MH, Koop SE, Novacheck TF. The identification and treatment of gait problems in cerebral palsy. Clinics in Developmental Medicine. 2009; No.180-18. 
[36] Kerr C, McDowell BC, Parkes J, Stevenson M, Cosgrove AP. Age-related changes in energy efficiency of gait, activity, and participation in children with cerebral palsy. Dev Med Child Neurol. 2011; 53(1): 61-67. doi: 10.1111/j.1469-8749.2010. 03795.x.

[37] Bell KJ, Ounpuu S, DeLuca PA, Romness MJ. Natural Progression of gait in children with cerebral palsy. J Pediatr Orthop. 2002; 22(5): 677-682.

[38] Verschuren O, Peterson MD, Balemans ACJ, Hurvitz EA Exercise and physical activity recommendations for people with cerebral palsy. Dev Med Child Neurol. 2016; 58(8): 798808. doi: 10.1111/dmen.13053.

[39] Ryan JM, Cassidy EE, Noorduyn SG, O'Connell NE. Exercise interventions for cerebral palsy. Cochrane Database Syst Rev. 2017; 6(6): CD011660. doi: 10.1002/14651858.CD 011660.pub2.

[40] Rogers A, Furler BL, Brinks S, Darrah J. A systematic review of the effectiveness of aerobic exercise interventions for children with cerebral palsy: An AACPDM evidence report. Dev Med Child Neurol. 2008; 50(11): 808-814. doi: 10.1111/j. 1469-8749.2008.03134.x.

[41] Lampe R, Grassl S, Mitternacht J, Gerdesmeyer L, Gradinger R. Measurements of muscle volumes of the lower extremities of youth with spastic hemiplegia caused by cerebral palsy. Brain Dev. 2006; 28(8): 500-506. doi: 10.1016/j.braindev. 2006.02.009.

[42] Ries AJ, Novacheck TF, Schwartz MH. The efficacy of anklefoot orthoses on improving the gait of children with diplegic cerebral palsy: A multiple outcome analysis. PM R. 2015; 7(9): 922-929. doi: 10.1016/j.pmrj.2015.03.005.

[43] Flanigan M, Gaebler-Spira D, Kocherginsky M, Garrett A, Marciniak C. Spasticity and pain in adults with cerebral palsy. Dev Med Child Neurol. 2020; 62(3): 379-385. doi: 10.1111/ dmcn. 14368 .

[44] Rosales RL, Bigalke H, Dressler D. Pharmacology of botulinum toxin: Differences between type A preparations. Eur J Neurol. 2006; 13: 2-10. doi: 10.1111/j.1468-1331.2006.01438. $\mathrm{x}$.
[45] Stackhouse SK, Binder-Macleod SA, Lee SCK. Voluntary muscle activation, contractile properties, and fatigability in children with and without cerebral palsy. Muscle Nerve. 2005; 31(5): 594-601. doi: 10.1002/mus.20302.

[46] Fairhurst C, Kumar R, Checketts D, Tayo B, Turner S. Efficacy and safety of nabiximols cannabinoid medicine for paediatric spasticity in cerebral palsy or traumatic brain injury: A randomized controlled trial. Dev Med Child Neurol. 2020; 62(9): 1031-1039. doi: 10.1111/dmcn.14548.

[47] Notcutt W, Langford R, Davies P, Ratcliffe S, Potts R. A placebo controlled parallel group randomized withdrawal study of subjects with symptoms of spasticity due to multiple sclerosis who are receiving long term Salivex (Nabiximols). Mult Scler. 2012; 18(2): 219-228. doi: 10.1177/1352458511419700.

[48] Heetla HW, Staal MJ, Kliphuis C, van Laar T. The incidence and management of tolerance in intrathecal baclofen therapy. Spinal Cord. 2009; 47(10): 751-756. doi: 10.1038/sc.2009.34

[49] Weiner WJ, Koller WC, Perlik S, Nausieda PA, Klawans HL Drug holiday and management of Parkinson disease. Neurology. 1980; 30(12): 1257-1261. doi: 10.1212/wnl.30.12.1257.

[50] Regnart J, McCartney J, Truter I. Drug holiday utilization in ADHD-diagnosed children and adolescents in South Africa. J Child Adolesc Ment Health. 2014; 26(2): 95-107. doi: 10.2989/ 17280583.2014.885905.

[51] Howland RH. Medication holidays. J Psychosoc Nurs Ment Health Serv. 2009; 47(9): 15-18. doi: 10.3928/02793695-200 90804-01.

[52] Moreau NG, Teefey SA, Damiano DL. In vivo muscle architecture and size of the rectus femoris and vastus lateralis in children and adolescents with cerebral palsy. Dev Med Child Neurol. 2009; 51(10): 800-806. doi: 10.1111/j.1469-8749.2009. 03307.x.

[53] Dai AI, Wasay M, Awan S. Botulinum toxin type A with oral baclofen versus oral tizanidine: A nonrandomized pilo comparison in patients with cerebral palsy and spastic equinus foot deformity. J Child Neurol. 2008; 23(12): 1464-1466. doi: 10.1177/0883073808319074. 